\title{
The evolution of stellar surface activity and possible effects on exoplanets
}

\author{
Mark S. Giampapa ${ }^{1}$ \\ ${ }^{1}$ National Solar Observatory/NOAO \\ 950 N. Cherry Ave., POB 26732, Tucson, Arizona USA 85726-6732 \\ email: giampapa@noao.edu
}

\begin{abstract}
The evolution of stellar activity involves a complex interplay between the interior dynamo mechanism, the emergent magnetic field configurations and their coupling with stellar winds, the subsequent angular momentum evolution, and fundamental stellar parameters. The discussion of the evolution of surface activity will emphasize the main sequence phase, from the ZAMS to stars of solar-age. We will focus particularly on the evolution of the fractional area coverages of spots on the surfaces of solar-type stars. We fit an empirical relation to the fractional mean spot area coverage as a function of age for ages greater than the Pleiades of the form $\log ($ MeanSpotCoverage $)=0.90( \pm 0.26)-1.03( \pm 0.10) \log ($ Age $)$, where Age is in Myr. In addition, we summarize the relative evolution of radiative emissions in various short wavelength bands that are associated with stellar magnetic field-related activity. Possible effects on young planetary atmospheres also are appropriate to consider given that stellar surface activity is the origin of the high-energy component of the ambient radiation and particle fields in which planetary atmosphere evolution occurs.
\end{abstract}

Keywords. Stars:spots, stars:activity, stars:planetary systems

\section{Introduction}

Spots and granulation are the most obvious inhomogeneities on the surface of the Sun. Spots are associated with strong magnetic fields and they are the most obvious manifestation of stellar surface 'activity', which we will define for the purposes of this discussion as the consequence of interactions of magnetic fields with the surrounding plasma. The granulation pattern is the result of outer convection. While we typically do not associate granulation with magnetic activity, the interaction of magnetic fields and granulation is seen in the form of systematic changes that are discernible through high spectral resolution observations of photospheric line profiles. For example, W. C. Livingston (National Solar Observatory) and collaborators find that the average amplitude of the natural asymmetry of absorption lines in the spectrum of the Sun-as-a-star, arising from the velocity-brightness correlation in upward moving granular cells, varies with the solar cycle. In particular, the asymmetry as measured by the mean bisector amplitude (see Gray 2008) is at a maximum near the time of solar minimum. It declines to a minimum near the time of solar maximum, consistent with what would occur if surface magnetic fields acted to reduce upflowing granulation velocities.

Stellar surface activity is exhibited in the spatially unresolved observations of stars primarily through line and continuum emission arising from chromospheres and coronae consisting of plasma that has been heated to temperatures well in excess of the stellar effective temperature. Brightness changes in the photometric light curves of late-type stars on rotational time scales, or longer time scales that are the stellar analogs of the 11-year sunspot cycle, also are associated with stellar surface features that are, in turn, delineated by magnetic fields. In the Sun, these brightness changes are seen in the variability of the 
Total Solar Irradiance (TSI) and the Spectral Solar Irradiance (SSI). Transient activity such as flare outbursts and Coronal Mass Ejections (CMEs), and irradiance changes, also may influence the evolution of planetary atmospheres, initially in young exoplanet systems and, later, affect planetary climates in more mature systems.

We will discuss the nature and apparent sources of irradiance and brightness changes seen in the Sun and sun-like stars, respectively, and estimate the form of the evolution of spot coverage on solar-type stars combined with a review of the evolution of coronal and chromospheric emission in late-type dwarfs. Potential effects on planetary atmospheres will be briefly discussed. Recent reviews of the evolution of stellar activity include Guinan \& Engle (2009); Engle, Guinan \& Mizusawa, T. (2009); and, Preibisch \& Feigelson (2005). The possible effects of host stars in an astrobiology context are discussed by Cuntz, Guinan \& Kurucz (2010). This is not an exhaustive list but these papers and references therein should enable the interested reader to pursue this topic in more depth.

\section{Convection, magnetic fields and irradiance variability}

Given the observation of solar irradiance variations in phase with the solar cycle, and in view of the $\sim 4$ million granules on the disk of the Sun at any given time, we might ask whether random variations in the number and size of turbulent cells combined with modulation of granule structures by the solar magnetic field could contribute to variations in heat flux. Conversely, in view of the modulation of the solar irradiance primarily due to magnetic structures such as faculae and spots, and the existence of modulation of stellar photometric light curves, also presumably by cool spots, we might justifiably ask why these brightness changes or irradiance variations are seen at all instead of the "blocked light" (in the case of spots) simply being re-radiated elsewhere.

Apparently, the answer lies in the enormous thermal inertia of the outer convection zone in the Sun and late-type stars. This same inertia tends to damp luminosity variations we might otherwise expect from structural changes deeper in the Sun. The light blocked by sunspots simply isn't re-radiated elsewhere on the Sun. Instead, the energy is stored in the convective envelope where it very slightly increases the potential and internal energy of the entire outer convection zone. The blocked heat flux remains stored in the Sun for hundreds of millennia instead of appearing elsewhere on the surface (Foukal et al. 2006). In faculae, the effect of their magnetic structure is to cause a slight localized depression in the photosphere, enabling radiation from lower and hotter layers to escape more easily. The predicted effects on changes in the solar radius are unobservably small.

Stellar 'Irradiance' Variability. Analogous to solar TSI is brightness variability in solartype stars as observed in selected photometric bands spanning the visible range. The same kind of correlated changes in brightness with variations in chromospheric Ca II $\mathrm{H}$ \& $\mathrm{K}$ core emission that are seen in the Sun during the course of the solar cycle also are observed in sun-like stars. However, unlike the Sun, anti-correlated brightness and chromospheric variability also is observed, as depicted in Fig. 1 from Radick et al. (1998).

Inspection of Fig. 1 reveals that the correlated and anti-correlated variability is associated with mean chromospheric emission level. The more active stars characterized by anti-correlated variability are typically younger, as can be seen from the superimposed 600 Myr Hyades isochrone. The correlation itself is thought to arise from the balance in fractional area coverage of bright facular features versus cool, dark spots so that in more active solar-type stars that are younger than the Sun, the net effect of the fractional area coverage of spots versus faculae is to produce a relative dimming with increasing chromospheric emission. Thus, a salient feature of the evolution of surface activity in sun-like stars is the change in the relative balance between dark spots and bright faculae 


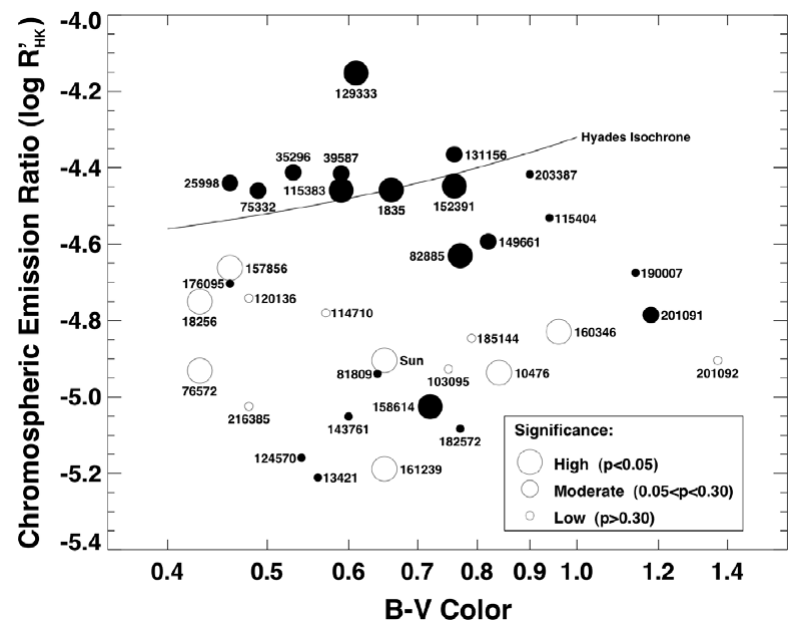

Figure 1. Correlated (open circles) and anti-correlated variability (filled circles) as a function of mean chromospheric emission level and color. Younger, more active stars exhibit anti-correlated variability while stars of solar-age with solar-like activity are characterized by variability that is directly correlated with chromospheric variations

in addition to the well known decline of the mean level of chromospheric emission with age.

\section{The evolution of star spots}

Using data from Messina (Rodonò \& Guinan), Radick et al. (1995) and Saar, Barnes \& Meibom (2010), I constructed Fig. 2 showing the logarithm of the 'Mean Spot Amplitude' as a function of age. The spot amplitude is really the amplitude of the photometric light curve, which is interpreted as the modulation of a star's V-band brightness by rotation carrying starspots across the line of sight. The vertical 'error bars' represent the onesigma dispersion of spot amplitudes in the cluster.

Inspection of Fig. 2 shows that in the young clusters ranging from $30 \mathrm{Myr}$ (IC 2391) to 100 Myr (Pleiades), spot amplitudes cluster around $\sim 10 \%$. There is a sharp, linear (in the $\log$ ) decline to around $1 \%$ by the age of the Hyades (600 Myr) and $\sim 0.1 \%$ $0.2 \%$ by the age of the Sun. However, there is a large gap in the available data at the intermediate age range between the Hyades and the Sun. Photometric monitoring of stars in an intermediate-age cluster such as NGC $752(\sim 2$ Gyr $)$ would be valuable in filling in this gap. Fitting the data in Fig. 2 with a linear function yields for the mean fractional area coverage of starspots at ages of the Pleiades or greater

$$
\log (\text { MeanSpotCoverage })=0.90( \pm 0.26)-1.03( \pm 0.10) \log (\text { Age }),
$$

where Age is in units of Myr. The above fitted relation can be rewritten to a good approximation simply as (Starspot Area) $\approx 10 /$ (Age), where, again, Age is in units of Myr.

The shape of the correlation in Fig. 2 is very much reminiscent of that for activity diagnostics as a function of rotation period, as shown in Fig. 3. The upper panel from Pizzolato et al. (2003) shows coronal X-ray luminosity as a function of rotation period (on a logarithmic scale) for individual objects. This radiative diagnostic can be compared to the lower graph of mean spot amplitude versus age. Of course, the extent of spots in the photospheres of solar-type and other late-type stars must be intimately related to 


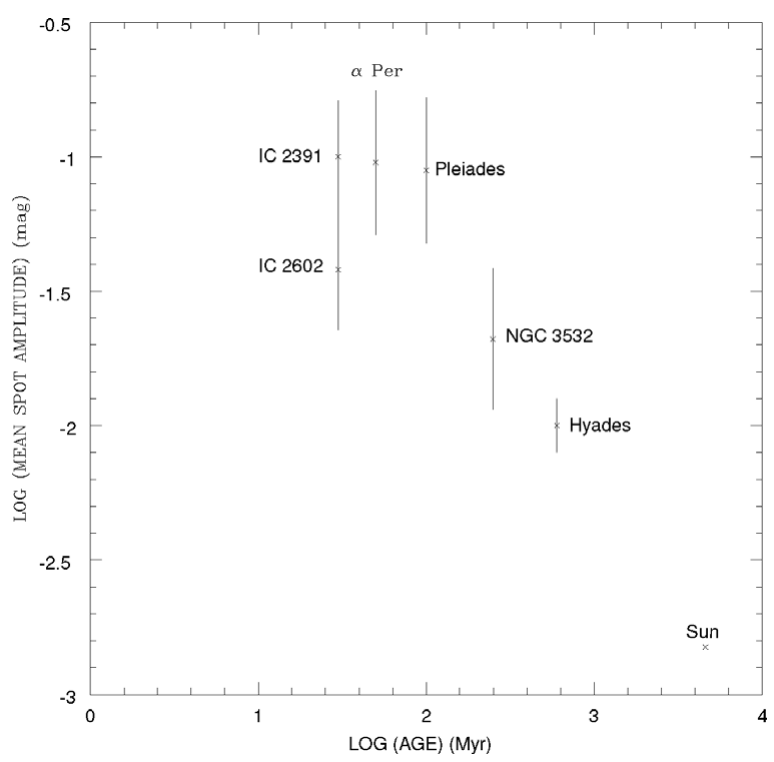

Figure 2. The evolution of the mean area coverage of star spots from the ages of young clusters to the age of the Sun. The vertical bars represent the one-sigma dispersion of spot amplitudes in the cluster

rotation via the internal dynamo. Stellar rotation rates are also a function of stellar age as a result of the interaction of stellar winds with extended magnetic field configurations combined with magnetic braking. The lower right panel in Fig. 3 shows the mean rotation period in the clusters and the Sun, and its correlation with age. The vertical 'error bars' represent the one-sigma dispersion in rotation periods for all the stars in their respective clusters with measured rotation periods, regardless of spectral type. Not surprisingly, the shape of this relation is very similar to the one for mean spot amplitude versus age. The similarity of the shape of the relations along with those of, say, $\mathrm{L}_{x}$ vs. rotation period, suggests that rotation determines total magnetic flux on stars, i.e., it is not just field strength that increases with rotation rates.

At this point we digress to note that the argument could be made that a decreasing amplitude of periodic variability in photometric light curves as a function of age could be interpreted as due to a more uniform spatial distribution of the same fractional area coverage of spots on the star as opposed to a real decrease in the total filling factor of spots. However, the concomitant decrease in mean levels of chromospheric and coronal emission along with the change from inversely to directly correlated brightness changes with chromospheric variability imply that the decline in photometric amplitudes with age is the result of a real decline in the filling factor of spots with stellar age.

Rotation rates decline more rapidly in the higher mass $G$ dwarfs than in the lower mass M dwarfs. Thoughts as to why this is the case include decoupled spin-down of the outer convection zone, different dominant magnetic field configurations or simply a decrease in mass loss rates toward lower mass stars on the main sequence. The associated chromospheric and coronal emission also decline with decreasing rotation, with coronal $\mathrm{X}$-ray emission depending sensitively on rotation and, hence, exhibiting a more rapid decline. The change (decline) in X-ray emission depends sensitively on rotation while lower-temperature chromospheric emission also decreases but not as rapidly. The rate of decline is more rapid at shorter wavelengths and becomes less rapid at UV wavelengths. It is these latter wavelengths that are more relevant to the photochemistry of the 

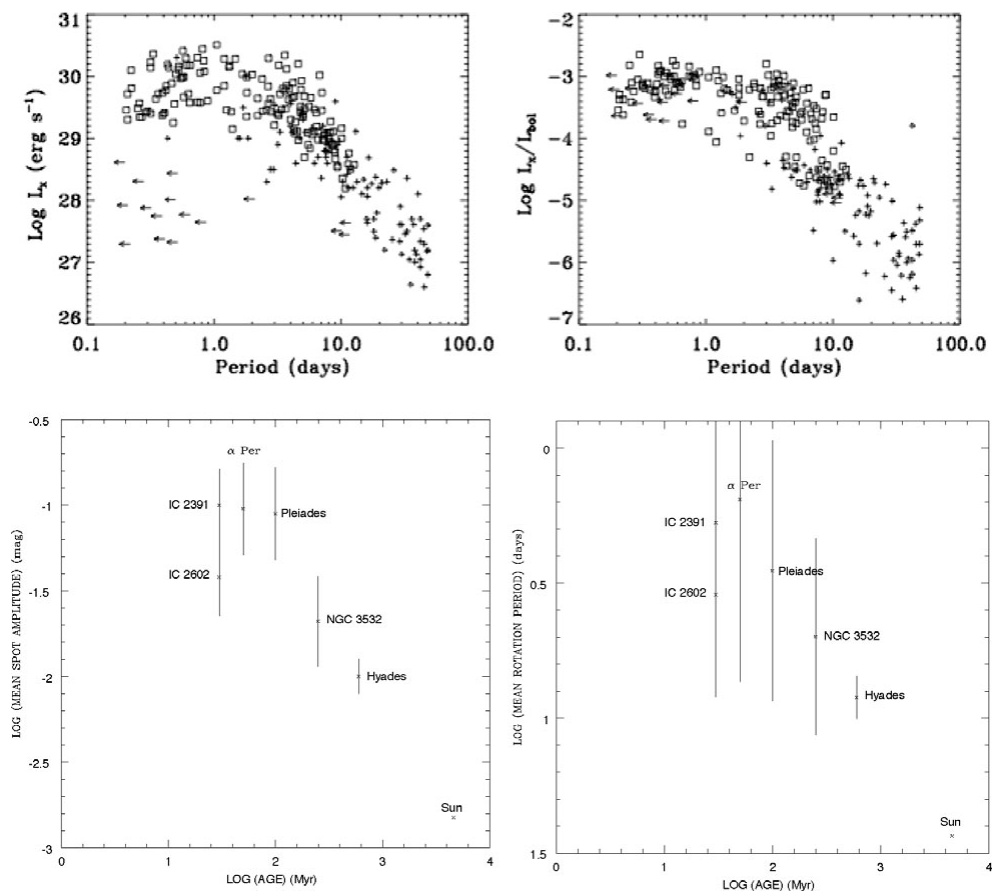

Figure 3. A comparison of the shapes of the mean spot fractional surface coverage versus age relation with the coronal activity and spot area coverage relations, respectively, each with rotation period. The similar shapes of these relations imply a common origin via the dynamo mechanism

atmospheres of planets, e.g., ozone chemistry is sensitive to UV emission. Hence, enhanced activity can have a more extended, longer-term influence in the UV compared to shorter wavelengths.

In this context, the recent study by Segura et al. (2010) merits a brief review. These authors examined the effect of a large UV flare event observed on the dM3.5e star, AD Leo, on an earth-like atmosphere for a planet located in the habitable zone at $\sim 0.1 \mathrm{AU}$ in a cool dwarf star of this type (Kaltenegger \& Traub 2009). Segura et al. also assumed that an energetic proton event analogous to those that occur in major solar flares was associated with the UV event. The planet was assumed not to have a magnetic field that could shield it from energetic particles. They found that the enhanced UV and energetic particles incident on the earth-like atmosphere in the habitable zone destroyed $\sim 90 \%$ of the ozone column for $\sim 10$ years before it recovered to its initial pre-flare state. The potential hazard for life is somewhat ambiguous because the pre-flare, 'quiescent' nearUV flux from this cool dwarf is relatively low compared to that of the Sun. However, it must be remembered that large flare events of this kind occur relatively frequently on a dwarf M flare star such as AD Leo. Moreover, evidence from X-ray light curves suggest that some degree of flaring is occurring continuously in this star (Giampapa et al. 1996) so that enhanced UV emission and energetic particle radiation likely characterizes the ambient radiative and particle environments in the habitable zones of active $M$ dwarf stars.

Finally, recent work suggests that the nature of the outer convection zone in latetype stars not only plays an important role in the operative dynamo mechanism, the emergence of magnetic flux and the formation of spots but that it also influences the orbital inclination, or 'obliquities', of exoplanets. In particular, Winn et al. (2010) claim 
that tidal dissipation through interaction between the exoplanet and the mass in the outer convection zone acts to dampen obliquities to near zero in solar-type and cooler dwarfs. Conversely, in warmer stars with thin, low-mass convection zones, exoplanet systems exhibit a wide range of obliquities.

In conclusion, the enormous yield of starspot rotational modulation results emerging from the ultra-high precision photometry from the Kepler mission will undoubtedly increase our understanding of the relationship between spot characteristics and stellar properties on rotational and evolutionary time scales. We can also look forward to the development of techniques such as Zeeman Doppler Imaging and high precision spectropolarimetry combined with advanced modeling techniques that, together, will enable us to carry out 'solar physics' on stars.

\section{References}

Cuntz, M., Guinan, E. F., \& Kurucz, R. L. 2010, Solar and Stellar Variability: Impact on Earth and Planets, Proceedings of the International Astronomical Union, IAU Symposium, 264, 419

Engle, S. G., Guinan, E. F., \& Mizusawa, T. 2009, Future Directions in Ultraviolet Spectroscopy: A Conference Inspired by the Accomplishments of the Far Ultraviolet Spectroscopic Explorer Mission, AIP Conf. Proc., 1135, p. 221

Foukal, P., Frölich, C., Spruit, H., \& Wigley, T. M. L. 2006, Nature, 443, 161

Giampapa, M. S., Rosner, R., Kashyap, V., Fleming, T. A., Schmitt, J.H.M.M., \& Bookbinder, J. A.,1996, Astrophys. J., 463, 707

Gray, D. F. 2008, The Observation and Analysis of Stellar Photospheres (Cambridge: Cambridge University Press)

Guinan, E. F \& Engle, S. G. 2009, The Ages of Stars, Proceedings of the International Astronomical Union, IAU Symposium, 258, 395

Kaltenegger, L. \& Traub, W. A. 2009, Astrophys. J., 698, 519

Livingston, W. C. 2010, private communication

Messina, S., Rodonò, M., \& Guinan, E. F. 2001, Astron. Astrophys, 366, 215

Pizzolato, N., Maggio, A., Micela, G., Sciortino, S., \& Ventura, P. 2003, Astron. Astrophys, 397, 147

Preibisch, T. \& Feigelson, E. D. 2005, Astrophys. J. Suppl., 160, 390

Radick, R. R., Lockwood, G. W., Skiff, B. A., \& Thompson, D. T. 1995, Astrophys. J., 452, 332

Radick, R. R., Lockwood, G. W., Skiff, B. A., \& Baliunas, S. L. 1998, Astrophys. J. Suppl., 118, 239

Saar, S. H., Barnes, S., \& Meibom, S. 2010, BAAS, 216, 833

Segura, A., Walkowicz, L., Meadows, V., Kasting, J., \& Hawley, S. 2010, Astrobiology, in press

Winn, J. N., Fabrycky, D., Albrecht, S., \& Johnson, J. A. 2010, Astrophys. J. Lett., 718, L145 